MS28-P03

\section{On Magnetic and Crystal Structures of NiO, MnO}

Pomjakushin Vladimir ${ }^{1}$

1. Lab. for Neutron Scattering, Paul Scherrer Institute, Villigen, Switzerland

email: vladimir.pomjakushin@psi.ch

Since the first neutron diffraction studies of manganese and nickel monoxides [1] the interest to the magnetic and crystal structures of $\mathrm{MnO}$ and $\mathrm{NiO}$ is kept up, see e.g. experimental study [2] and theoretical first principal calculations [3] and references cited therein. It is well established experimentally that below $\mathrm{N}^{\prime}$ eel temperature the crystal structure is metrically lowered in symmetry from cubic $\mathrm{F} \overline{\mathrm{m}} 3 \mathrm{~m}$ to rhombohedral $\overline{\mathrm{R}} 3 \mathrm{~m}$, or might be to monoclinic one. However, the allowed by symmetry monoclinic distortions from rhombohedral $\overline{\mathrm{R}} 3 \mathrm{~m}$-metric are very small and the explicit monoclinic splittings of the diffraction peaks have not been experimentally observed. We analyse in full details all possible magnetic crystallographic models metrically compatible with the experiment by using isotropy subgroup representation approach based on the propagation vector star. The models (some not previously considered) are compared with the high resolution and high intensity diffraction data measured at the HRPT diffractometer at Swiss neutron spallation source SINQ.

References:

[1] C. G. Shull, W. A. Strauser, and E. O. Wollan, (1951), Physical Review 83, 333,

[2] S. Lee, Y. Ishikawa, P. Miao, S. Torii, T. Ishigaki, and T. Kamiyama, (2016) Physical Review B 93064429.

[3] J. S. Lim, D. Saldana-Greco, and A. M. Rappe, (2016) Physical Review B 94, 165151.

Keywords: magnetic, structure, symmetry,

\section{MS28-P04}

\section{Crystal chemistry features and physical properties of three Mn phosphates}

Larisa Shvanskaya $^{1}$, Olga Yakubovich ${ }^{1}$, Galina Kiriukhina ${ }^{1}$, Alexander Vasiliev ${ }^{1}$

1. M.V. Lomonosov Moscow State University, Moscow, Russia email: lshvanskaya@mail.ru

The complex phosphates with manganese cations in low oxidation state $\left(\mathrm{Mn}^{2+}\right)$ attract attention as potential catalysts for industrially important oxygen reduction reactions [1]. For example, $\mathrm{Mn}_{3}\left(\mathrm{PO}_{4}\right)_{2} * 3 \mathrm{H}_{2} \mathrm{O}$ are found to display interesting electrocatalytic properties for water splitting [2]. Besides catalytic activity, Mn-based phosphates can demonstrate rare magnetic grounds states, which make these compounds attractive both for experimental physical property investigation and for theoretical calculation.

Three manganese phosphates, namely $\mathrm{RbMn}_{4}\left(\mathrm{PO}_{4}\right)_{3}(\mathrm{I})$, $\mathrm{CsMnPO}_{4}(\mathrm{II})$ and $\mathrm{Mn}_{2} \mathrm{PO}_{4} \mathrm{OH}(\mathrm{III})$ were studied by single crystal X-ray diffraction and characterized by measuring of the magnetic susceptibility, magnetization, and heat capacity. The $\mathrm{RbMn}_{4}\left(\mathrm{PO}_{4}\right)_{3}$ belongs to the morphotropic series of oxo-salts with general formula $A M_{4}\left(T \mathrm{O}_{4}\right)_{3}$. The $\mathrm{Mn}_{2} \mathrm{PO}_{4} \mathrm{OH}$ presents a manganese end-member of the triplite-triploidite family. The (I,III) crystal structures can be described as three-dimensional frameworks of corner and edge-sharing $\mathrm{MnO}_{5}$ and $\mathrm{MnO}_{6}$ polyhedra which are further straightened by $\mathrm{PO}_{4}$ tetrahedra. $\mathrm{CsMnPO}_{4}$ presents a novel polymorphic modification with zeolite $\mathrm{ABW}$-type framework consisting of tridimite-like sheets of six-membered rings of manganese and phosphorus tetrahedra sharing vertices. The magnetic ground states of (I) and (II) are canted antiferromagnet below $\mathrm{T}_{\mathrm{N}}=3.1 \mathrm{~K}$ and $\mathrm{T}_{\mathrm{N}}=4.7 \mathrm{~K}$, respectively. The $\mathrm{Mn}_{2} \mathrm{PO}_{4} \mathrm{OH}$ compound orders antiferromagnetically at $\mathrm{T}_{\mathrm{N}}=4.6 \mathrm{~K}$. In general, the magnetic behavior of $\mathrm{CsMnPO}_{4}$ is similar to that of structurally related $\mathrm{RbMnPO}_{4}$ [3]. Despite three dimensional magnetic structures of $\mathrm{RbMn}_{4}\left(\mathrm{PO}_{4}\right)_{3}$ and Mn${ }_{2} \mathrm{PO}_{4} \mathrm{OH}$, both phosphates demonstrate low dimensional magnetic behavior. Their specific feature is the high ratio of Weiss and Neel temperatures $|\Theta| / T_{N}$, called frustration parameter. This value is equal to 10 for $\mathrm{RbMn}_{4}\left(\mathrm{PO}_{4}\right)_{3}$ and is twice as high for $\mathrm{Mn}_{2} \mathrm{PO}_{4} \mathrm{OH}$. The origin of high frustration degree in the second compound is associated with the twisted saw tooth chain geometry of corner sharing triangles of manganese polyhedra (Fig.1, left), which may be isolated within tubular fragments of its crystal structure. The chain of another topology (Fig.1, right) is seemingly responsible for significant frustration in $\mathrm{RbMn}_{4}\left(\mathrm{PO}_{4}\right)_{3}$. The finite magnetization of $\mathrm{RbMn}_{4}\left(\mathrm{PO}_{4}\right)_{3}$ observed for $\mathrm{T}<\mathrm{T}_{\mathrm{N}}$ might be explained by a Dzyaloshinskii-Moriya interaction.

Our investigation led us to establish the relation between the crystal structure and thermodynamic properties.

The work was supported by the Russian Foundation for Basic Research (grant No. 18-03-00908).

References:

[1] Zhan, Y. M. et al. (2016). ChemCatChem. 8, 372 - 379 .

[2] Jin, K. et al. (2014). J. Am. Chem. Soc. 136, 7435-7443.

[3] Nénert, et al. (2013). Inorg. Chem. 52, 9627-9635.

Keywords: phosphates, crystal chemistry, antiferromagnet 